\title{
Comment on: "Clomiphene Citrate co-treatment with low dose urinary FSH versus urinary FSH for clomiphene resistant PCOS: randomized controlled trial." by Ghanem et al.
}

\author{
A. Papathanasiou
}

Received: 19 September 2013 / Accepted: 9 October 2013 / Published online: 26 October 2013

(C) Springer Science+Business Media New York 2013

Dear Editor,

I read with great interest the recently published randomized controlled trial entitled "Clomiphene Citrate co-treatment with low dose urinary FSH versus urinary FSH for clomiphene resistant PCOS" by Ghanem et al., which deals with the challenging group of anovulatory PCOS women who are resistant to standard clomiphene treatment [1]. I would like to raise some concerns regarding the methodology and result reporting in the study, which, I believe, have a significant bearing on the interpretation of its findings.

Within their flowchart, the authors reported the numbers of women who ovulated within each group (63 women in the study group and 33 women in the control group). These numbers do not match the ones reported in the main table of results (72 women in the per cycle result in the study group, 30 women in the per woman result $/ 24$ women in the per cycle result in the control group). These inconsistencies not only produce invalid statistics, but also reduce the validity of the study in general. Although applying the corrected values may still reveal significant differences in ovulation rates between the two groups, the size of the effect will also be different from the reported one. Publication of accurate data is crucial for all studies but more so for experimental trials, whose data are frequently pooled in subsequently published meta-analyses.

Apart from the experimental part of the study, the authors also reported an analysis of independent predictors for

\footnotetext{
A. Papathanasiou $(\bowtie)$

Department of Health Sciences, University of Aberdeen, Foresterhill, Aberdeen AB25 2ZL, UK

e-mail: a.papathanasiou@abdn.ac.uk
}

ovulation from the same dataset of patients. After comparing the characteristics between the ovulatory and anovulatory groups, they performed ROC analysis for every characteristic that was different between these groups and reported that the treatment protocol used was the only independent predictor of ovulation. It is confusing to the reader that the authors report a $95 \% \mathrm{CI}$ for the ROC curve which is significant $(0.51-0.77)$, while the reported $\mathrm{p}$-value is non-significant $(p=0.05)$. One could also argue that the number of available cases (174 women) is less than optimal for performing an informative prediction analysis and this is promptly reflected in the wide confidence intervals of the ROC analysis. Furthermore, ROC analysis does not adjust for confounders in the way multivariate analysis does and, thus, should not be preferred for prediction modelling. Identifying the treatment protocol as the only predictor for ovulation does not add to the findings. This research question has already been answered by the experimental part of the study.

I would like to thank the authors of the study for providing clinicians with a useful alternative treatment protocol of ovulation induction in clomiphene resistant PCOS women.

\section{References}

1. Ghanem ME, Elboghdady LA, Hassan M, Helal AS, Gibreel A, Houssen M, Shaker ME, Bahlol I, Mesbah Y. Clomiphene Citrate co-treatment with low dose urinary FSH versus urinary FSH for clomiphene resistant PCOS: randomized controlled trial. J Assist Reprod Genet. 2013 Sep 7. (Epub ahead of print) 